\title{
Nano-porous Zeolite and MOF Filled Mixed Matrix Membranes for Gas Separation
}

\author{
Yongsheng LIU, Kyosuke TAKATA, Yu MUKAI, Hidetoshi KITA and Kazuhiro TANAKA* \\ Graduate School of Sciences and Technology for Innovation, Yamaguchi University, 2-16-1 Tokiwadai, Ube-shi, Yamaguchi 755- \\ 8611, Japan
}

\begin{abstract}
The commercial SAPO-34 zeolite with $0.38 \mathrm{~nm}$ pore size and ZIF-8 particles with 0.34 $\mathrm{nm}$ aperture size were separately dispersed into different polymer matrix, to prepare the mixed matrix membranes (MMMs) for gas separation. The dispersed situation of the SAPO-34 and ZIF8 particles in matrix and the influence of the fillers on the separation performance of the membrane had been investigated in this study. The as-synthesized MMMs showed a better trade-off between permeability and selectivity than the pure polymer membrane and the performance could exceed or close to the upper bound line of polymer membrane for $\mathrm{CO}_{2}$ and $\mathrm{CH}_{4}$ separation. The $\mathrm{CO}_{2}$ permeability and $\mathrm{CO}_{2} / \mathrm{CH}_{4}$ ideal selectivity of the 6FDA-mDAT MMM containing $40 \mathrm{wt} \%$ SAPO-34 zeolite was 190 barrer and ca. 60, respectively. The 6FDA-TrMPD based MMMs containing 20 wt $\%$ ZIF-8 provided a permeability of $\mathrm{C}_{3} \mathrm{H}_{6}$ and an ideal selectivity of $\mathrm{C}_{3} \mathrm{H}_{6} / \mathrm{C}_{3} \mathrm{H}_{8}$ at 24 barrer and ca. 17, respectively. These separation performances were in a suitable agreement of the theoretical value from Maxwell model.
\end{abstract}

\section{Introduction}

Gas separation technologies are crucial for several industrial processes, including $\mathrm{CO}_{2}$ capture and storage (CCS), and olefin/paraffin separation. Carbon dioxide widely exists in the exhaust gas and result in the greenhouse effect. Besides, the $\mathrm{CO}_{2}$ acts as an acid gas impurity of the natural gas could corrode the transport pipeline and reduce the heating value. The U.S. Energy Information Administration had reported that the consumption market of nature gas was 27.85 trillion cubic feet (TCF) in 2017, and it will up to 31.91 TCF in 2040. Propylene is the basic material of three major synthetic materials. According to a reference, the world demand of propene was 114 million tons in 2015 and has growing at a rate of 5\% annually (Christopher, et al., 2017). The urgent demand for CCS and propene production promotes the development and application of the related separation materials and technologies (Mondal, et al., 2012; Zhou, et al., 2018).

The 4,4'(hexafluoroisopropylidene)-diphthalic anhydride (6FDA) based aromatic polyimide (PI) membranes could provide a high gas permeability and have been widely investigated for $\mathrm{CO}_{2} / \mathrm{CH}_{4}$ and $\mathrm{C}_{3} \mathrm{H}_{6} / \mathrm{C}_{3} \mathrm{H}_{8}$ separation (Favvas, et al., 2017; Swaidan, et al., 2015). The 6FDA-2,6-diaminotoluene-(6FDAmDAT) and the 6FDA-2,4,6-trimethyl-1,3phenylenediamine (6FDA-TrMPD) have a similar backbone, while the former has only one methyl in each monomer molecule and the latter has three. The number of the methyl groups could control the stiffness of their backbone (Tanaka, et al., 1992; Bachman, et al., 2016). However, polymer membranes always reach a limit in trade-off between permeability and selectivity for gas separation. Robeson revealed an upper bound line of polymer membranes based on the above phenomenon (Robeson, 2008). In the recently reports, an effective improve method was blending the porous inorganic filler particles into polymer matrix to fabricate a new membrane, the so-called mixed matrix membranes (MMMs). It could combine both advantages of the polymer membrane and the inorganic membrane (Cheng, et al., 2018).

In terms of the filler choice, silicoaluminophosphate-34 (SAPO-34) zeolite is an interesting candidate because of its rigid pore size ca. $0.38 \mathrm{~nm}$ and high adsorption affinity to $\mathrm{CO}_{2}$ (Funke, et al., 2014). There are some literatures reported that the SAPO-34 improved the separation properties of the polymer membrane. For example, the $\mathrm{CO}_{2}$ permeability and $\mathrm{CO}_{2} / \mathrm{CH}_{4}$ selectivity of PES membrane could be increased by $22 \%$ and $13 \%$ through adding 20\% SAPO-34 (Wu, et al., 2019). Commercial Matrimid ${ }^{\circledR} 5218$ was blended with 20 wt $\%$ SAPO-34, its $\mathrm{CO}_{2}$ permeability and $\mathrm{CO}_{2} / \mathrm{CH}_{4}$ selectivity was increased by $55 \%$ and $103 \%$ (Peydayesh, et al., 2013). To our best knowledge, there are few researches about adding SAPO34 zeolite into the 6FDA-mDAT and 6FDA-TrMPD matrix, especially using the commercial filler.

Another promising filler, ZIF-8, has also been widely researched for MMMs preparation, because it has an aperture size ca. $0.34 \mathrm{~nm}$. Besides, its excellent intrinsic organic moiety provides a better compatibility of

\footnotetext{
* Corresponding author: tnkkzhr@yamaguchi-u.ac.jp
} 
polymeric matrix than other inorganic filler. Others prior properties, such as large pore volume and flexible framework are also important for improving the performance of the membrane. Up to now, Matrimid/ZIF8 (Carter, et al., 2017), P48/ZIF-8 (Guo, et al., 2018) and others ZIF-8 filled MMMs (Dechnik, et al., 2017) had been fabricated, the properties of the pure polymer membrane had been improved. According to some reports, the ZIF-8 was already incorporated in the polyimides, and could obviously enhance the separation performance of the neat polymer membrane for $\mathrm{CO}_{2} / \mathrm{CH}_{4}$ and $\mathrm{C}_{3} \mathrm{H}_{6} / \mathrm{C}_{3} \mathrm{H}_{8}$ mixture (Carter, et al., 2017; Zhang, et al., 2012a).

Herein, we would try to discuss the dispersed situation and the influence of the SAPO-34 and ZIF-8 particles in the 6FDA-mDAT and 6FDA-TrMPD polymer matrix. The polyethersulfone (PES), which has a better flexibility backbone than the 6FDA based polyimides, was also used as a polymer matrix in this study.

\section{Experimental}

\subsection{Materials}

The monomers 6FDA (99 wt\%), TrMPD (98 wt $\%$ ) mDAT (98 wt $\%$ ) were purchased from TCI company. Acetic anhydride (97 wt \%), triethylamine (99 wt\%) and the solvent N-methylpyrrolidone (NMP, $99 \mathrm{wt} \%$ ) were purchased from Wako company. The ZIF-8 powder

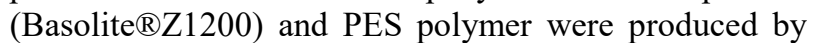
BASF Chemical Co. (Germany). The SAPO-34 powder was provided by Nikki Universal Co., Ltd.

\subsection{Synthesis of polyimide}

The polyimide polymer (6FDA-TrMPD and 6FDAmDAT) was synthesized from their monomers. The polyamic acid was synthesized in nitrogen atmosphere. Firstly, the diamine monomer, 6FDA monomer, acetic acid, triethylamine and solvent NMP were mixed in a four-neck round-bottomed flask. The catalyst acetic anhydride and triethylamine added into the mixture when it became a clear solution. The molar ratio of diamine monomer: 6FDA: acetic anhydride: triethylamine was 1: 1: $4: 4$. The imidization was carried out at $50^{\circ} \mathrm{C}$ for 2 hours. The last solution was poured into ethanol and washed for 3 times. The washed polymer was dried at $80^{\circ} \mathrm{C}$ overnight.

\subsection{Preparation of membranes}

The initial ZIF-8 powder was treated by ball milling at $120 \mathrm{rpm}$ for three days to eliminate the agglomeration effect of small particles. The filler particles were mixed with NMP for pre-wetting treatment and then the dried polymer added in the solution. After ultrasonic treatment for 4 hours and bubble removal for 30 minutes, the solution was cast on the surface of a glass plate, and then dried at $200^{\circ} \mathrm{C}$ for 20 hours. The film was peeled from the glass plate. The neat polymer membrane was prepared with the same method except no filler particles added. The abbreviation method of the as-synthesized membrane as following: the 6FDA-mDAT/SAPO-34 MMMs were presented by M-S-x, $\mathrm{x}$ was the weight ratio of filler in polymer from 0 to 40 (wt $\%$ ); 6FDA-TrMPD/SAPO-34 MMMs were abbreviated in T-S-x; 6FDA-TrMPD/ZIF-8 MMMs were named T-Z-x; the P-S-x were the PES/SAPO-34 MMMs.

\subsection{Characterization methods}

The X-ray diffraction (XRD, Rigaku Smartlab 9/SWXD) was used to analysis the structure of the MMMs. Scanning electron microscopy (SEM, JEOL, JSM 6335F) could be applied to observe the membranes' morphology.

The single gas permeation of $\mathrm{CO}_{2}$ and $\mathrm{CH}_{4}$ was carried out in a home-made equipment with a vacuum method. The feed pressure and temperature were $1 \mathrm{~atm}$ and $35^{\circ} \mathrm{C}$, respectively. The permeation of $\mathrm{C}_{3} \mathrm{H}_{6}$ and $\mathrm{C}_{3} \mathrm{H}_{8}$ through the 6FDA-TrMPD/ZIF-8 MMMs was at 1 atm and $35-100^{\circ} \mathrm{C}$. The permeability coefficient $(\mathrm{P})$ and ideal selectivity $(\alpha)$ were calculated by the following equations.

$$
\begin{aligned}
& P=\frac{Q l}{A \Delta p} \\
& \alpha=\frac{P_{i}}{P_{j}}
\end{aligned}
$$

Where the $Q$ was the volume flow rate of the gas at the standard temperature and pressure (STP); $l$ was the membrane thickness; $A$ was the effective area of the membrane; $\Delta p$ was the pressure drop; $i$ and $j$ were the permeating molecules. The unit of permeability coefficient $P$ was barrer ( 1 barrer $=10^{-10} \mathrm{~cm}^{3}(\mathrm{STP}) \mathrm{cm}$ $\left.\mathrm{cm}^{-2} \mathrm{~s}^{-1} \mathrm{cmHg}^{-1}\right)$.

\section{Results and Discussion}

\subsection{XRD characterization}
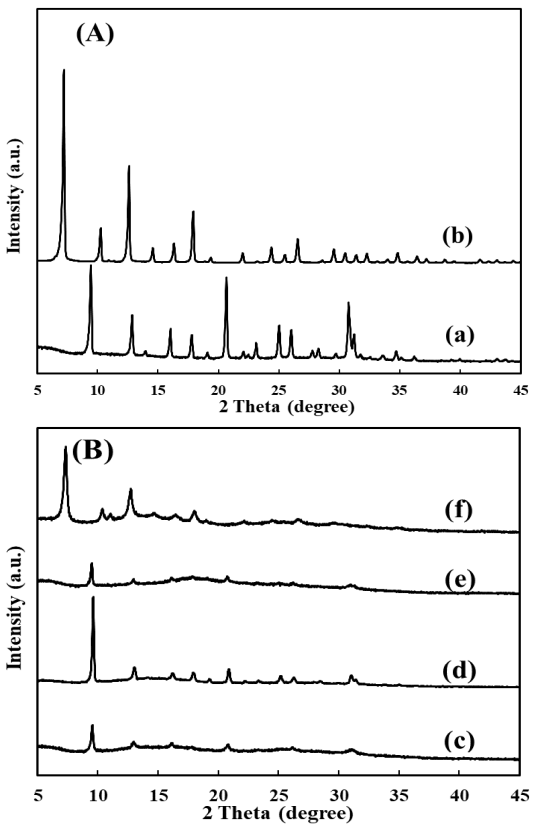

Figure 1. XRD patterns of (A) initial filler powder and (B) the as-synthesized MMMs: (a) SAPO-34 initial powder; (b) ZIF-8 initial powder; (c) M-S-40; (d) T-S-40; (e) P-S-30; (f) T-Z-40. 
The XRD patterns of two filler powder were displayed in the Figure 1. The zeolite powder possessed a typical XRD peaks belong to CHA structure. Another powder also shown the XRD peaks in according to the ZIF-8 structure. The XRD patterns of 30\% SAPO-34 filled PES MMM (PS-30), 40\% SAPO-34 filled 6FDA-TrMPD MMMs (T-S40), the 6FDA-mDAT MMMs containing 40\% SAPO-34 (M-S-40) and 40\% ZIF-8 filled 6FDA-TrMPD MMM (TZ-40) were also shown in the Figure 1. The characteristic peaks of the filler appeared in each MMMs, even if the initial ZIF-8 powder treated by ball milling.

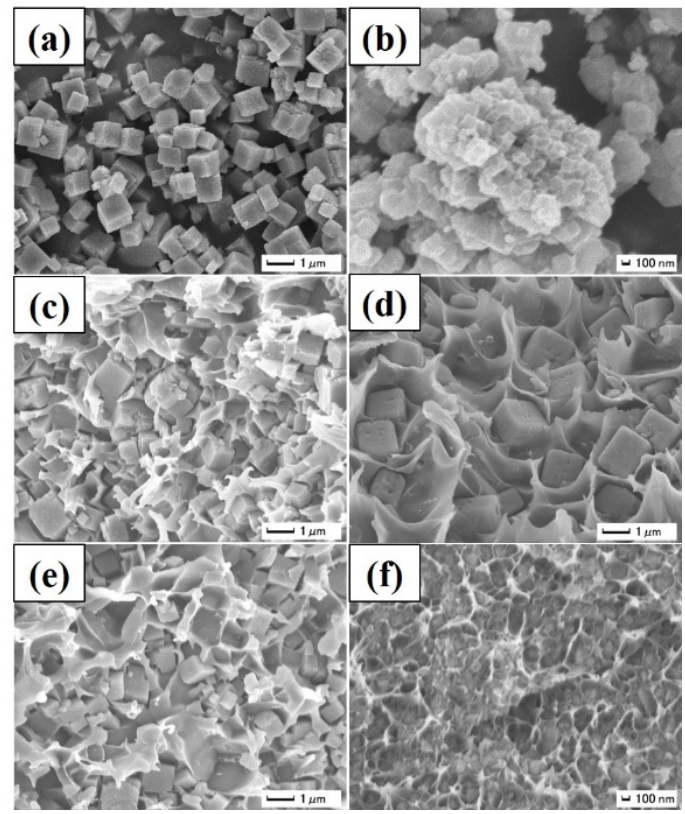

Figure 2. SEM images of initial filler powder and the crosssection of the as-synthesized MMMs: (a) SAPO-34 initial powder; (b) ZIF-8 initial powder; (c) M-S-40; (d) T-S-40; (e) P-S-30; (f) T-Z-40.

\subsection{SEM characterization}

According to the Figure 2a, the zeolite powder has the typical cubic crystals, the particle size was around $1 \mu \mathrm{m}$. The MOF powder in Figure $2 b$ also showed a cubic shape with the particle size around $200 \mathrm{~nm}$. The nano-sized particles easily agglomerate. The agglomerate had been eliminated by ball milling method, as shown in the Figure 2f. The ball-milled ZIF-8 particles also had a smaller size than initial particle. It contributed to the distribution of ZIF-8 particles in the polymer matrix and reduce the interfacial defect between the filler and the matrix.

The SAPO-34 zeolite particles were homogeneously dispersed into three kinds of polymer matrix based on the cross-sectional SEM images (Figure 2). Unfortunately, some sieve-in-a-cages could be observed from each crosssection of the membranes. Their appearance was due to the poor compatibility between inorganic zeolite filler and polymer matrix. The affinity of the filler and the matrix depended on the nature properties of filler and matrix. The 6FDA-TrMPD possessed a rigid backbone so that its based MMM had the largest amounts of sieve-in-a-cages compared with 6FDA-mDAT and PES based MMMs.

\subsection{Gas separation performance}

Maxwell model could be used to describe the gas transport though the mixed matrix membrane (Zhang, et al., 2012a). To investigate the role of SAPO-34 and ZIF8 , the comparison of the theoretical value from Maxwell model and empirical value from experiment was made in this study. The calculation method by Maxwell model as following:

$$
P=P_{1} \times \frac{P_{1}+2 P_{2}-2 v\left(P_{1}-P_{2}\right)}{P_{2}+2 P_{1}+v\left(P_{1}-P_{2}\right)}
$$

Where $P, P_{1}$ and $P_{2}$ was the gas permeability of MMMs, pure polymer matrix (continuous phase) and pure filler (dispersed phase); $v$ was the volume fraction of the filler particles. The separation performance of the neat polymer was from this study. The $\mathrm{CO}_{2}$ permeability and $\mathrm{CO}_{2} / \mathrm{CH}_{4}$ selectivity of pure SAPO-34 membrane were 2090 barrer and $100\left(\mathrm{Wu}\right.$, et al., 2015). The $\mathrm{C}_{3} \mathrm{H}_{6}$ permeability and $\mathrm{C}_{3} \mathrm{H}_{6} / \mathrm{C}_{3} \mathrm{H}_{8}$ ideal selectivity of the pure ZIF-8 membrane were 210 barrer and 84 (Zhang, et. al., 2012b).

The theoretical value calculated by Maxwell equation for each system was represented in a black solid circle in the Figure 3. In each graph, the filler content of the solid circles from left to right was $0 \mathrm{wt} \%, 10 \mathrm{wt} \%, 20 \mathrm{wt} \%, 30$ $\mathrm{wt} \%, 40 \mathrm{wt} \%$ and $50 \mathrm{wt} \%$, respectively.

\subsubsection{SAPO-34 filled MMMs}

According to the Figure 3a, adding $40 \mathrm{wt} \%$ SAPO-34 zeolite could obviously increase the $\mathrm{CO}_{2}$ permeability as well as the $\mathrm{CO}_{2} / \mathrm{CH}_{4}$ ideal selectivity, comparing with the neat 6FDA-mDAT membrane (M-S-0). The improvement was benefit from the suit pore size of the SAPO-34 (0.38 $\mathrm{nm})$. The separation performance of the M-S-40 exceeded the Robeson upper line of the polymeric (2008). It was also overlapping with the theoretical value predicted by Maxwell model at $30 \mathrm{wt} \%$ filler content and closing to the theoretical value at $40 \mathrm{wt} \%$.

However, there was a different change trend on the separation performance of 6FDA-TrMPD based membrane after adding the SAPO-34 zeolite, as shown in Figure $3 \mathrm{~b}$. Increasing the SAPO-34 content from $0 \mathrm{wt} \%$ (T-S-0) from $40 \mathrm{wt} \%$ (T-S-40) could only enhance the $\mathrm{CO}_{2}$ gas permeability, but not improve the ideal selectivity of $\mathrm{CO}_{2} / \mathrm{CH}_{4}$. The existence of the sieve-in-acages or other defects was considered to result in the unexpected performance. There was a competition effect during the sieve-in-a-cages and SAPO-34 zeolite pore for gas transport, the former even provided a route for $\mathrm{CH}_{4}$ easier to transport. It would increase both the permeability of $\mathrm{CO}_{2}$ and $\mathrm{CH}_{4}$ and decreased gas selectivity of $\mathrm{CO}_{2} / \mathrm{CH}_{4}$. While the experimental result displayed that the selectivity of $\mathrm{CO}_{2} / \mathrm{CH}_{4}$ remained a relatively stable. The $\mathrm{Al}$ or $\mathrm{P}$ atoms which located in SAPO-34 zeolite framework are in favour of the adsorption of $\mathrm{CO}_{2}$ (Funke, et al., 2014). It could offset some part of the negative influence from sieve-in-a-cages. The amount of sieve-ina-cage of M-S-40 (Figure 2c) was less than T-S-40 (Figure 2d) also suggested the improvement of the separation performance of the former could be better than of the latter. Nevertheless, the separation performance of 
T-S-30 and T-S-40 exceeded the Robeson upper bound (2008) as well as the M-S-40.
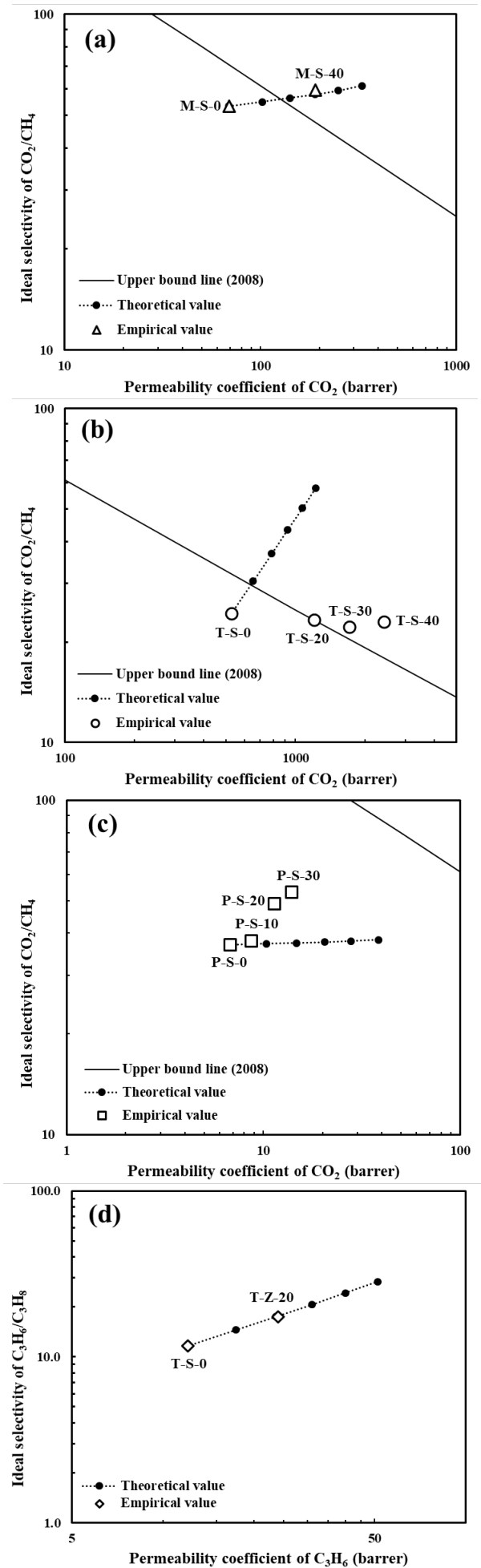

Figure 3. Comparison of theoretical value and empirical value on the performance of the as-synthesized MMMs with different filler content for $(\mathrm{a}-\mathrm{c}) \mathrm{CO}_{2} / \mathrm{CH}_{4}$ and $(\mathrm{d})$ $\mathrm{C}_{3} \mathrm{H}_{6} / \mathrm{C}_{3} \mathrm{H}_{8}$ separation at $1 \mathrm{~atm}$ and $35^{\circ} \mathrm{C}$ : (a) $6 \mathrm{FDA}-$ mDAT/SAPO-34 MMMs; (b) 6FDA-TrMPD/SAPO34 MMMs; (c) PES/SAPO-34 MMMs; (d) 6FDATrMPD/ZIF-8 MMMs. In each graph, the filler content of the solid circles from left to right was $0 \%$, $10 \%, 20 \%, 30 \%, 40 \%$ and $50 \%$, respectively.
Due to the PES possessed the best flexibility of the polymer chain compared with the 6FDA-mDAT and 6FDA-TrMPD, the PES had the best affinity with the SAPO-34 zeolite filler. It could reduce the interfacial defects. In this case, the $\mathrm{CO}_{2} / \mathrm{CH}_{4}$ ideal selectivity could be increased with the SAPO-34 content increasing (Figure $3 \mathrm{c})$. Adding $40 \mathrm{wt} \% \mathrm{SAPO}-34$ led to the $\mathrm{CO}_{2}$ permeability and $\mathrm{CO}_{2} / \mathrm{CH}_{4}$ selectivity increased by ca. $100 \%$ and $44 \%$, respectively. The ideal selectivity of the as-synthesized PES/SAPO-34 MMMs were all higher than the theoretical value by Maxwell model.

\subsubsection{ZIF-8 filled MMMs}

The ball-milled ZIF-8 nano-particles had also been used to improve the performance of 6FDA-TrMPD membrane for separating $\mathrm{CO}_{2}$ and $\mathrm{CH}_{4}$. Even if no sieve-in-a-cage could be clearly observed from the SEM image (Figure 2f) of the T-Z-40 membrane, the $\mathrm{CO}_{2} / \mathrm{CH}_{4}$ selectivity still decreased by ca. $30 \%$ compared with the neat film. A literature reported that ZIF- 8 has a flexible structure, the pore size could up to $0.42 \mathrm{~nm}$ (Fairen-Jimenez, et al., 2011). Zhang, et al., (2012b) reported that the pure ZIF-8 membrane only provided a selectivity of $\mathrm{CO}_{2} / \mathrm{CH}_{4}$ about 12, which less than of neat film. Hence, it was reasonable that the gas $\mathrm{CO}_{2}$ permeability of 6FDA-TrMPD increased at loss of selectivity of $\mathrm{CO}_{2} / \mathrm{CH}_{4}$ after adding ZIF- 8 in this study.

In order to identify the absence of the defect and further explore the role of ZIF-8, the $\mathrm{C}_{3} \mathrm{H}_{6}$ with a kinetic diameter about $0.4 \mathrm{~nm}$ and $\mathrm{C}_{3} \mathrm{H}_{8}$ with a kinetic diameter ca. $0.42 \mathrm{~nm}$ could be used as the probe molecules in this study. After adding $20 \mathrm{wt} \% \mathrm{ZIF}-8$, the $\mathrm{C}_{3} \mathrm{H}_{6}$ permeability and $\mathrm{C}_{3} \mathrm{H}_{6} / \mathrm{C}_{3} \mathrm{H}_{8}$ ideal selectivity of the neat film were both improved. The separation performance was highly consistent with the theoretical value. It confirmed that ZIF-8 performed well in the MMMs for $\mathrm{C}_{3} \mathrm{H}_{6} / \mathrm{C}_{3} \mathrm{H}_{8}$ separation.

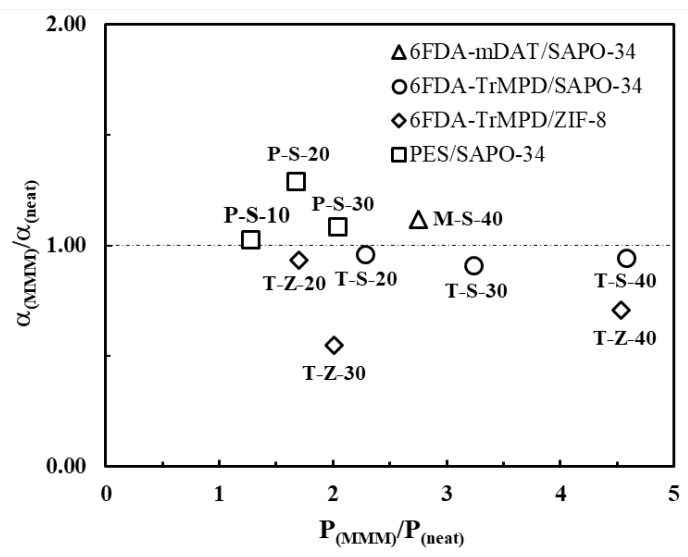

Figure 4. Scatter diagram of the $\mathrm{CO}_{2}$ permeability ratio and $\mathrm{CO}_{2} / \mathrm{CH}_{4}$ selectivity ratio of the as-synthesized MMMs and the neat film at $1 \mathrm{~atm}$ and $35^{\circ} \mathrm{C}$.

The scatter diagram about permeability ratio and ideal selectivity ratio of the MMMs and neat film could make a summary about the influence of the fillers on the polymer matrix. In terms of $\mathrm{CO}_{2} / \mathrm{CH}_{4}$ separation, the SAPO-34 zeolite provided the best improvement on the PES based membrane (Figure 4). The SAPO-34 particles almost 
synchronously enhance the permeability and selectivity of the 6FDA-TrMPD based membrane.

\section{Conclusions}

For $\mathrm{CO}_{2} / \mathrm{CH}_{4}$ separation, the SAPO-34 zeolite could both enhance the gas permeability and selectivity of 6FDAmDAT based membranes and PES based membranes. The compatibility of the filler and polymer matrix influenced their MMMs' separation performance. The flexible structure of ZIF-8 particles acted as some nonselective pathways for $\mathrm{CO}_{2}$ and $\mathrm{CH}_{4}$, while it could benefit the large gas molecules separation. The separation performance of 6FDA-mDAT MMM containing $40 \mathrm{wt} \%$ SAPO-34 for $\mathrm{CO}_{2} / \mathrm{CH}_{4}$ and 6FDA-TrMPD MMM containing $20 \mathrm{wt} \%$ ZIF-8 for $\mathrm{C}_{3} \mathrm{H}_{6} / \mathrm{C}_{3} \mathrm{H}_{8}$ was in a suitable agreement with the theoretical value from Maxwell model.

\section{References}

Bachman, J. and J. Long; "Plasticization-resistant $\mathrm{Ni}_{2}$ (dobdc)/Polyimide Composite Membranes for the Removal of $\mathrm{CO}_{2}$ from Natural Gas," Energy Environ. Sci., 9, 2031-2036 (2016)

Carter, D., F. H. Tezel, B. Kruczek, and H. Kalipcilar; "Investigation and Comparison of Mixed Matrix Membranes Composed of Polyimide Matrimid with ZIF8, Silicalite, and SAPO-34," J. Membr. Sci., 544, 35-46 (2017)

Cheng, Y., Z. Wang, and D. Zhao; "Mixed Matrix Membranes for Matural Gas Upgrading: Current Status and Opportunities," Ind. Eng. Chem. Res., 57, 4139-4169 (2018)

Christopher, C., A. Dutta, S. Farooq, and I. Karimi; "Process Synthesis and Optimization of Propylene/Propane Separation Using Vapor Recompression and Self-heat Recuperation," Ind. Eng. Chem. Res., 56, 14557-14564 (2017)

Dechnik, J., C. J. Sumby, and C. Janiak; "Enhancing Mixed-Matrix Membrane Performance with MetalOrganic Framework Additives," Cryst. Growth Des., 17, 4467-4488 (2017)

Fairen-Jimenez, D., S. A. Moggach, M. T. Wharmby, P. A. Wright, S. Parosons, and T. Düren; "Opening the Gate: Framework Flexibility in ZIF-8 Explored by Experiments and Simulations," J. Am. Chem. Soc., 133, 8900-8902 (2011)

Favvas, E. P., F. K. Katsaro, S. K. Papageogiou, A. A. Sapalidis, and A. C. Mitropoulos; "A Review of the Latest Development of Polyimide Based Membranes for $\mathrm{CO}_{2}$ Separations," React. Funct. Polym., 120, 104-130 (2017)

Funke, H. H., M. Z. Chen, A. N. Prakash, J. L. Falconer, and R. D. Noble; "Separating Molecules by Size in
SAPO-34 Membranes," J. Membr. Sci., 456, 185-191 (2014)

Guo, A., Y. Ban, K. Yang, and W. Yang; "Metal-organic Framework-based Mixed Matrix Membranes: Synergetic Effect of Adsorption and Diffusion for $\mathrm{CO}_{2} / \mathrm{CH}_{4}$ Separation," J. Membr. Sci., 562, 76-84 (2018)

Mondal, M. K., H. Balsora, and P. Varshney; "Progress and Trends in $\mathrm{CO}_{2}$ Capture/Separation Technologies: A review," Energy, 46, 431-441 (2012)

Peydayesh, M., S. Asarehpour, T. Mohammadi, and O. Bakhtiari; "Preparation and Characterization of SAPO34-Matrimid®5218 Mixed Matrix Membranes for $\mathrm{CO}_{2} / \mathrm{CH}_{4}$ Separation," Chem. Eng. Res. Des., 91, 1335-1342 (2013)

Robeson, L. M.; "The Upper Bound Revisited," J. Membr. Sci., 320, 390-400 (2008)

Swaidan, R., X. Ma, E. Litwiller, and I. Pinnau; "Enhanced Propylene/Propane Separation by Thermal Annealing of an Intrinsically Microporous HydroxylFunctionalized Polyimide Membrane," J. Membr. Sci., 495, 235-241 (2015)

Tanaka, K., M. Okano, H. Toshino, H. Kita, and K. I. Okamoto; "Effect of Methyl Substituents on Permeability and Permselectivity of Gases in Polyimides Prepared from Methyl-substituted Phenylenediamines," J. Polym. Sci., Part B: Polymer. Phys., 30, 907-914 (1992)

Wu, T., M. C. Diaz, Y. Zheng, R. Zhou, H. H. Funke, J. L. Falconer, and R. D. Noble; "Influence of Propane on $\mathrm{CO}_{2} / \mathrm{CH}_{4}$ and $\mathrm{N}_{2} / \mathrm{CH}_{4}$ Separations in CHA Zeolite Membranes," J. Membr. Sci., 473, 201-209 (2015)

Wu, T., Y. Liu, I. Kumakiri, K. Tanaka, X. Chen, and H. Kita; "Preparation and Permeation Properties of PESUbased Mixed Matrix Membranes with Nano-sized CHA zeolites," J. Chem. Eng. Japan, 52, 514-520 (2019)

Zhang, C., Y. Dai, J. R. Johnson, O. Karvan, and W. J. Koros; "High Performance ZIF-8/6FDA-DAM Mixed Matrix Membrane for Propylene/Propane Separations," $J$. Membr. Sci., 389, 34-42 (2012a)

Zhang, C., R. P. Lively, K. Zhang, J. R. Johnson, O. Karvan, and W. J. Koros; "Unexpected Molecular Sieving Properties of Zeolitic Imidazolate Framework-8," J. Phys. Chem. Lett., 3, 2130-2134 (2012b)

Zhou, S., Y. Wei, L. Li, Y. Duan, Q. Hou, L. Zhang, L. Ding, J. Xue, H. Wang, and J. Caro; "Paralyzed Membrane: Current-driven Synthesis of a Metal-organic Framework with Sharpened Propene/Propane Separation," Sci. Adv., 4, eaau1393 (2018) 